\title{
Painlevé analysis of the coupled nonlinear Schrödinger equation for polarized optical waves in an isotropic medium
}

\author{
Q-Han Park* and H. J. Shin ${ }^{\dagger}$ \\ Department of Physics \\ and \\ Research Institute of Basic Sciences \\ Kyunghee University, Seoul, 130-701, Korea
}

\begin{abstract}
Using the Painlevé analysis, we investigate the integrability properties of a system of two coupled nonlinear Schrödinger equations that describe the propagation of orthogonally polarized optical waves in an isotropic medium. Besides the well-known integrable vector nonlinear Schrödinger equation, we show that there exist a new set of equations passing the Painlevé test where the self and cross phase modulational terms are of different magnitude. We introduce the Hirota bilinearization and the Bäcklund transformation to obtain soliton solutions and prove integrability by making a change of variables. The conditions on the third-order susceptibility tensor $\chi^{(3)}$ imposed by these new integrable equations are explained.
\end{abstract}

\section{INTRODUCTION}

The coupling between copropagating optical pulses in a nonlinear medium has led to many important applications in optical fiber systems such as optical switching and soliton-dragging logic gates [1]. The governing equation for the propagation of two orthogonally polarized pulses in a monomode birefringent fiber is given by the coupled nonlinear Schrödinger(NLS) equation, where the nonlinear coupling terms are determined by the third-order susceptibility tensor $\chi^{(3)}$ of the fiber. In an isotropic medium, the tensor $\chi^{(3)}$ has three independent components $\chi_{x x y y}^{(3)}, \chi_{x y x y}^{(3)}$ and $\chi_{x y y x}^{(3)}$ and the nonlinear polarization components which account for the nonlinear coupling terms take the form

$$
\begin{aligned}
& P_{x}=\frac{3 \epsilon_{0}}{2}\left[\left[\left(\chi_{x x y y}^{(3)}+\chi_{x y x y}^{(3)}+\chi_{x y y x}^{(3)}\right)\left|E_{x}\right|^{2}+\left(\chi_{x x y y}^{(3)}+\chi_{x y x y}^{(3)}\right)\left|E_{y}\right|^{2}\right] E_{x}+\chi_{x y y x}^{(3)} E_{y}^{2} E_{x}^{*}\right], \\
& P_{y}=\frac{3 \epsilon_{0}}{2}\left[\left[\left(\chi_{x x y y}^{(3)}+\chi_{x y x y}^{(3)}+\chi_{x y y x}^{(3)}\right)\left|E_{y}\right|^{2}+\left(\chi_{x x y y}^{(3)}+\chi_{x y x y}^{(3)}\right)\left|E_{x}\right|^{2}\right] E_{y}+\chi_{x y y x}^{(3)} E_{x}^{2} E_{y}^{*}\right] .
\end{aligned}
$$

In the case of silicar fibers, $\chi_{x x y y}^{(3)} \approx \chi_{x y x y}^{(3)} \approx \chi_{x y y x}^{(3)}$ and the nonlinear terms above have a ratio of 3;2;1. However, when the fiber is elliptically birefringent with the ellipticity angle $\theta \approx 35^{\circ}$, and also the beat length due to birefrigence is much smaller than the typical propagation distances, the coupled NLS equation takes the form of the vector NLS equation whose nonlinear terms have a ratio of 1;1;0 [2], which is known to be integrable via the inverse scattering method [3] 四. In general, the coupled NLS equations with arbitrary coefficients are not integrable. Mathematically, there exists a systematic way of generalizing the NLS equation to the multi-component cases [5] and to the higherorder cases [6] using group theory which preserves the integrabiiity structure. This gives rise to various integrable, coupled NLS equations among $N$ scalar fields $\psi_{i} ; i=1, \ldots, N$ with specific set of coupling parameters. For $N=2$, the vector NLS equation is the only nontrivial integrable equation in the group theoretic construction. However, it is not known whether there can be other cases of the integrable coupled NLS equation for $N=2$ with nonlinear coupling terms as in Eq. (11) except for the vector NLS equation.

In this paper, using the Painlevé analysis we investigate the integrability properties of the coupled NLS equation relevant to the propagation of orthogonally polarized optical waves in an isotropic medium. Motivated by Eq. (1), we consider the general form of the coupled NLS equation such that

$$
\begin{aligned}
& i \bar{\partial} q_{1}=\partial^{2} q_{1}+q_{1}\left(\gamma_{1}\left|q_{1}\right|^{2}+\gamma_{2}\left|q_{2}\right|^{2}\right)+\gamma_{3} q_{1}^{*} q_{2}^{2}+\gamma_{4} q_{1}^{2} q_{2}^{*}, \\
& i \bar{\partial} q_{2}=\beta \partial^{2} q_{2}+q_{2}\left(\gamma_{2}\left|q_{1}\right|^{2}+\gamma_{1}\left|q_{2}\right|^{2}\right)+\gamma_{3} q_{2}^{*} q_{1}^{2}+\gamma_{4} q_{2}^{2} q_{1}^{*},
\end{aligned}
$$

*Electronic address; qpark@nms.kyunghee.ac.kr

${ }^{\dagger}$ Electronic address; hjshin@nms.kyunghee.ac.kr 
where $\beta= \pm 1$ signify the relative sign of the group-velocity dispersion terms and we use the notation $\partial=\partial / \partial z, \bar{\partial}=$ $\partial / \partial \bar{z}$. We find that the system passes the Painlevé test whenever the parameters belong to one of the following four classes; ( $i) \beta=1, \gamma_{1}=\gamma_{2}, \gamma_{3}=\gamma_{4}=0$, (ii) $\beta=1, \gamma_{2}=2 \gamma_{1}, \gamma_{3}=-\gamma_{1}, \gamma_{4}$ arbitrary , (iii) $\beta=1, \gamma_{2}=2 \gamma_{1}, \gamma_{3}=$ $\gamma_{1}, \gamma_{4}=0$ and (iv) $\beta=-1, \gamma_{1}=-\gamma_{2}, \gamma_{3}=\gamma_{4}=0$. Case (i)( and (iv)) is the well-known vector NLS equation. The integrability of cases (i) and (iv) have been demonstrated by Zakharov and Schulman by deriving an appropriate inverse scattering formalism [- [4] [7. However, cases (ii) and (iii) are new as far as we know. In particular, case (ii) corresponds to the propagation in the isotropic nonlinear medium with the property that $\chi_{x x y y}^{(3)}+\chi_{x y x y}^{(3)}=-2 \chi_{x y y x}^{(3)}$. We find the Hirota bilinearization and the Bäcklund transformation of cases (ii) and (iii), and compute soliton solutions. As for the integrability of cases (ii) and (iii), we prove that they are essentially identical to two independent NLS equations. This implies that in the case (ii), there is no physical interactions between two optical pulses with opposite circular polarizations. We also show that our Painlevé analysis is consistent with the group theoretical method of generalizing the integrable NLS equations when the group theoretical method is combined with the reduction procedure.

\section{PAINLEVÉ ANALYSIS OF THE COUPLED NLS EQUATION}

The Painlevé analysis for a partial differential equation was first introduced by Weiss, Tabor, and Carnevale [8] who defined that a partial differential equation has the Painlevé property if its general solution is single-valued about the movable singularity manifold. This method is to seek a solution of a given differential equation in a series expansion in terms of $\phi(z, \bar{z})=z-\psi(\bar{z})$, where $\psi(\bar{z})$ is an arbitrary analytic function of $\bar{z}$ and $\phi=0$ defines a non-characteristic movable singularity manifold. Then, the equation has the Painlevé property, thus becomes integrable, if there exists a sufficient number of arbitrary functions in the series solution. For $\beta=1$, we postulate a solution of the form,

$$
\begin{aligned}
& q_{1}=\sum_{m \geq 0} R_{m}(\bar{z})(z-\psi)^{m-\sigma}, \\
& q_{1}^{*}=\sum_{m \geq 0} S_{m}(\bar{z})(z-\psi)^{m-\sigma}, \\
& q_{2}=\sum_{m \geq 0} T_{m}(\bar{z})(z-\psi)^{m-\sigma}, \\
& q_{2}^{*}=\sum_{m \geq 0} U_{m}(\bar{z})(z-\psi)^{m-\sigma} .
\end{aligned}
$$

Substituting these ansätze into Eq. (2) and looking at the leading order behavior, we find that $\sigma=1$ and the following equations should be satisfied:

$$
\begin{gathered}
\gamma_{1} U_{0}^{2} T_{0}+\gamma_{2} R_{0} S_{0} U_{0}+\gamma_{3} S_{0}^{2} T_{0}+\gamma_{4} U_{0}^{2} R_{0}+2 U_{0}=0, \\
\gamma_{1} T_{0}^{2} U_{0}+\gamma_{2} R_{0} S_{0} T_{0}+\gamma_{3} R_{0}^{2} U_{0}+\gamma_{4} T_{0}^{2} S_{0}+2 T_{0}=0, \\
\gamma_{1} R_{0}^{2} S_{0}+\gamma_{2} R_{0} T_{0} U_{0}+\gamma_{3} T_{0}^{2} S_{0}+\gamma_{4} R_{0}^{2} U_{0}+2 R_{0}=0, \\
\gamma_{1} S_{0}^{2} R_{0}+\gamma_{2} S_{0} T_{0} U_{0}+\gamma_{3} U_{0}^{2} R_{0}+\gamma_{4} S_{0}^{2} T_{0}+2 S_{0}=0 .
\end{gathered}
$$

In order to facilitate solving Eq. (4), we define $x \equiv U_{0} R_{0}, y \equiv T_{0} S_{0}, t \equiv R_{0} S_{0}, s \equiv U_{0} T_{0}$ so that the first two equations in Eq. (4) can be written as

$$
\begin{aligned}
& \gamma_{1} s+\gamma_{2} t+2+\gamma_{4} x=-\gamma_{3} \frac{t y}{x}, \\
& \gamma_{1} s+\gamma_{2} t+2+\gamma_{4} y=-\gamma_{3} \frac{t x}{y},
\end{aligned}
$$

while the last two as

$$
\begin{aligned}
\gamma_{1} t+\gamma_{2} s+2+\gamma_{4} x & =-\gamma_{3} \frac{s y}{x}, \\
\gamma_{1} t+\gamma_{2} s+2+\gamma_{4} y & =-\gamma_{3} \frac{s x}{y} .
\end{aligned}
$$

Each pair can be combined to give $(x-y)\left(\gamma_{4}-\gamma_{3} t \frac{x+y}{x y}\right)=0$, and $(x-y)\left(\gamma_{4}-\gamma_{3} s \frac{x+y}{x y}\right)=0$. One can readily check that solutions of these equations can be classified in seven different cases,

(case 1) $x=y, \gamma_{1}=\gamma_{2}+\gamma_{3}$

(case 2) $x=y, t=x$, 
(case 3) $x=y, t=-x$,

(case 4) $t=s, \gamma_{4}=\gamma_{3} t \frac{x+y}{x y}$

(case 5) $x=-y, \gamma_{4}=0, \gamma_{2}=\gamma_{1}+\gamma_{3}, t+s=-2 / \gamma_{1}$

(case 6) $\gamma_{3}=\gamma_{4}=0, s=t=-2 /\left(\gamma_{1}+\gamma_{2}\right)$.

(case 7) $\gamma_{3}=\gamma_{4}=0, \gamma_{1}=\gamma_{2}, t+s=-2$

For each cases, we check the powers, so called resonances, at which the arbitrary functions can arise in the series solution. Equating coefficients of the $(z-\psi)^{j-3}$ term in Eq. (2) with the ansätze in Eq. (3), we obtain a system of four linear algebraic equations in $\left(R_{j}, S_{j}, T_{j}, U_{j}\right)$ which are given in a matrix form by,

$$
Q_{j}\left(\begin{array}{c}
R_{j} \\
S_{j} \\
T_{j} \\
U_{j}
\end{array}\right)=\left(\begin{array}{c}
F_{j} \\
G_{j} \\
H_{j} \\
K_{j}
\end{array}\right)
$$

The $4 \times 4$ matrix $Q_{j}=(j-1)(j-2) I_{4 \times 4}+\left(\begin{array}{cc}Q_{j}^{(1)} & Q_{j}^{(2)} \\ Q_{j}^{(3)} & Q_{j}^{(4)}\end{array}\right)$ has block components:

$$
\begin{aligned}
Q_{j}^{(1)} & =\left(\begin{array}{cc}
2 \gamma_{1} R_{0} S_{0}+\gamma_{2} T_{0} U_{0}+2 \gamma_{4} R_{0} U_{0} & \gamma_{1} R_{0}^{2}+\gamma_{3} T_{0}^{2} \\
\gamma_{1} S_{0}^{2}+\gamma_{3} U_{0}^{2} & 2 \gamma_{1} R_{0} S_{0}+\gamma_{2} T_{0} U_{0}+2 \gamma_{4} S_{0} T_{0}
\end{array}\right), \\
Q_{j}^{(2)} & =\left(\begin{array}{cc}
\gamma_{2} R_{0} U_{0}+2 \gamma_{3} T_{0} S_{0} & \gamma_{2} R_{0} T_{0}+\gamma_{4} R_{0}^{2} \\
\gamma_{2} S_{0} U_{0}+\gamma_{4} S_{0}^{2} & \gamma_{2} S_{0} T_{0}+2 \gamma_{3} U_{0} R_{0}
\end{array}\right), \\
Q_{j}^{(3)} & =\left(\begin{array}{cc}
\gamma_{2} T_{0} S_{0}+2 \gamma_{3} R_{0} U_{0} & \gamma_{2} R_{0} T_{0}+\gamma_{4} T_{0}^{2} \\
\gamma_{2} U_{0} S_{0}+\gamma_{4} U_{0}^{2} & \gamma_{2} R_{0} U_{0}+2 \gamma_{3} T_{0} S_{0}
\end{array}\right), \\
Q_{j}^{(4)} & =\left(\begin{array}{cc}
2 \gamma_{1} T_{0} U_{0}+\gamma_{2} R_{0} S_{0}+2 \gamma_{4} S_{0} T_{0} & \gamma_{1} T_{0}^{2}+\gamma_{3} R_{0}^{2} \\
\gamma_{1} U_{0}^{2}+\gamma_{3} S_{0}^{2} & 2 \gamma_{1} T_{0} U_{0}+\gamma_{2} R_{0} S_{0}+2 \gamma_{4} U_{0} R_{0}
\end{array}\right),
\end{aligned}
$$

and

$$
\begin{aligned}
F_{j} & \equiv-\sum_{0 \leq l, m, n<j}^{l+m+n=j}\left(\gamma_{1} R_{l} R_{m} S_{n}+\gamma_{2} R_{l} T_{m} U_{n}+\gamma_{3} S_{l} T_{m} T_{n}+\gamma_{4} R_{l} R_{m} U_{n}\right)+i R_{j-2}^{\prime}-i(j-2) \psi^{\prime} R_{j-1}, \\
G_{j} & \equiv-\sum_{0 \leq l, m, n<j}^{l+m+n=j}\left(\gamma_{1} S_{l} S_{m} R_{n}+\gamma_{2} S_{l} U_{m} T_{n}+\gamma_{3} R_{l} U_{m} U_{n}+\gamma_{4} S_{l} S_{m} T_{n}\right)-i S_{j-2}^{\prime}+i(j-2) \psi^{\prime} S_{j-1}, \\
H_{j} & \equiv-\sum_{0 \leq l, m, n<j}^{l+m+n=j}\left(\gamma_{1} T_{l} T_{m} U_{n}+\gamma_{2} T_{l} R_{m} S_{n}+\gamma_{3} U_{l} R_{m} R_{n}+\gamma_{4} T_{l} T_{m} S_{n}\right)+i T_{j-2}^{\prime}-i(j-2) \psi^{\prime} T_{j-1}, \\
K_{j} & \equiv-\sum_{0 \leq l, m, n<j}^{l+m+n=j}\left(\gamma_{1} U_{l} U_{m} T_{n}+\gamma_{2} U_{l} S_{m} R_{n}+\gamma_{3} T_{l} S_{m} S_{n}+\gamma_{4} U_{l} U_{m} R_{n}\right)-i U_{j-2}^{\prime}+i(j-2) \psi^{\prime} U_{j-1}
\end{aligned}
$$

The resonances occur when $\operatorname{det} Q_{j}=0$. Now, we compute the resonance values and check the Painlevé property of Eq. (2) for each seven cases as introduced above.

Case 1. $\mathbf{x}=\mathbf{y} ; \gamma_{1}=\gamma_{2}+\gamma_{3}$

In this case, we can solve for $T_{0}, R_{0}$ such that

$$
T_{0}=\frac{-2 U_{0}}{\gamma_{1}\left(S_{0}^{2}+U_{0}^{2}\right)+\gamma_{4} S_{0} U_{0}}, \quad R_{0}=\frac{-2 S_{0}}{\gamma_{1}\left(S_{0}^{2}+U_{0}^{2}\right)+\gamma_{4} S_{0} U_{0}} .
$$

When we substitute these solutions into the resonance condition, $\operatorname{det} Q_{j}=0$, we find that the resonances do not occur at the integer values of $j$. Therefore, this case does not pass the Painlevé test for integrability. 
Case 2 and Case 3. $\mathrm{x}=\mathrm{y} ; \mathrm{t}= \pm \mathrm{x}$

We have solutions,

$$
S_{0}= \pm U_{0}=\frac{-2}{\gamma_{1}+\gamma_{2}+\gamma_{3} \pm \gamma_{4}} \frac{1}{R_{0}}, \quad T_{0}= \pm R_{0}
$$

where + and - sign correspond to the Case 2 and the Case 3 respectively. Substituting these solutions into the resonance condition $\operatorname{det} Q_{j}=0$, we find that the resonance values $j=-1,0,1,1,2,2,3,4$ occur when $\gamma_{2}=2 \gamma_{1}, \gamma_{3}=$ $\pm \gamma_{4}+\gamma_{1}$. The resonance $j=-1$ is related with the arbitrariness of $\psi$, while the resonance $j=0$ is related with the arbitrariness of $R_{0}$. The recursion relation in Eq. (7) determines $R_{1}, S_{1}, T_{1}, U_{1}$ in terms of $R_{0}, S_{0}, T_{0}, U_{0}, \psi$. The degree of multiplicity of the resonance $j=1$ is two and it turns out that there exist two arbitrary functions consistently only if $\gamma_{4}=0$. Therefore, the case where $\gamma_{2}=2 \gamma_{1}, \gamma_{3}=\gamma_{1}$ and $\gamma_{4}=0$ passes the Painlevé test.

Case 4. $\mathbf{t}=\mathbf{s} ; \gamma_{4} \mathbf{x y}=\gamma_{\mathbf{3}} \mathbf{t}(\mathbf{x}+\mathbf{y})$

Eq. (4) together with the condition $t=s, \gamma_{4} x y=\gamma_{3} t(x+y)$ results in

$$
t=\frac{-2}{\gamma_{1}+\gamma_{2}-\gamma_{3}+\left(\gamma_{4}^{2} / \gamma_{3}\right)}, \quad x=\left(\frac{\gamma_{4}}{2 \gamma_{3}} \pm \sqrt{\left(\gamma_{4} / 2 \gamma_{3}\right)^{2}-1}\right) t
$$

and

$$
S_{0}=\frac{t^{2}}{x} \frac{1}{T_{0}}, \quad U_{0}=\frac{t}{T_{0}}, \quad R_{0}=\frac{x}{t} T_{0} .
$$

When we substitute these solutions into the resonance condition $\operatorname{det} Q_{j}=0$, we obtain

$$
(j-4)(j-3) j(j+1)\left(j^{2}-3 j+2 \frac{\gamma_{4}^{2}-4 \gamma_{3}^{2}}{\gamma_{4}^{2}-\gamma_{3}^{2}+\gamma_{2} \gamma_{3}+\gamma_{3} \gamma_{1}}\right)\left(j^{2}-3 j+2 \frac{\gamma_{4}^{2}-2 \gamma_{3}^{2}+2 \gamma_{2} \gamma_{3}-2 \gamma_{1} \gamma_{3}}{\gamma_{4}^{2}-\gamma_{3}^{2}+\gamma_{2} \gamma_{3}+\gamma_{1} \gamma_{3}}\right)=0 .
$$

Note that the Painlevé test requires the resonances $j$ to be integers and the degeneracy of resonance at $j=0$ to be one since there is only one arbitrary function $T_{0}$ as in Eq. (13). This requirement leads to the result, $\gamma_{2}=2 \gamma_{1}, \gamma_{3}=-\gamma_{1}$ and $\gamma_{4}$ arbitrary, so that resonances are $j=-1,0,1,1,2,2,3,4$. The recursion relation in Eq. (7) determines $T_{1}, U_{1}, T_{2}, U_{2}$ such as

$$
\begin{aligned}
T_{1} & =\frac{1}{4}\left(\sqrt{\gamma_{4}^{2}-4}-\gamma_{4}\right)\left(2 R_{1}+i \sqrt{\gamma_{4}^{2}-4} T_{0} \psi_{x}\right), \\
U_{1} & =-\frac{1}{2}\left(\sqrt{\gamma_{4}^{2}-4}+\gamma_{4}\right)\left(S_{1}+\frac{i}{\sqrt{\gamma_{4}^{2}-4}} \frac{\psi_{x}}{T_{0}}\right), \\
T_{2} & =\frac{1}{2}\left(\sqrt{\gamma_{4}^{2}-4}-\gamma_{4}\right)\left(R_{2}+\frac{\sqrt{\gamma_{4}^{2}-4}}{12}\left(T_{0} \psi_{x}^{2}+2 i \frac{\partial T_{0}}{\partial x}\right)\right), \\
U_{2} & =\frac{1}{12}\left(\sqrt{\gamma_{4}^{2}-4}+\gamma_{4}\right)\left(-6 S_{2}+\frac{i}{\sqrt{\gamma_{4}^{2}-4}}\left(\frac{\psi_{x}^{2}}{T_{0}}+2 i \frac{1}{T_{0}^{2}} \frac{\partial T_{0}}{\partial x}\right)\right) .
\end{aligned}
$$

Similarly, $R_{3}, T_{3}, U_{3}$ are determined in terms of $\psi, T_{0}, R_{1}, S_{1}, R_{2}, S_{2}$. In the same way, we can check that there exists one arbitrary function at the $\mathrm{j}=4$ resonance and no more arbitrary functions in higher lebels. All these facts have been confirmed with the symbolic manipulation program Macsyma. Thus, the system passes the Painlevé test when $\gamma_{2}=2 \gamma_{1}, \gamma_{3}=-\gamma_{1}$ and $\gamma_{4}$ arbitrary. We show that this case is indeed integrable in Sec. 3 .

Case 5. $\mathbf{x}=-\mathbf{y} ; \gamma_{\mathbf{4}}=\mathbf{0} ; \gamma_{\mathbf{2}}=\gamma_{\mathbf{1}}+\gamma_{\mathbf{3}} ; \mathbf{t}+\mathbf{s}=-\mathbf{2} / \gamma_{\mathbf{1}}$

In this case, the resonances are at $j=-1,0,0,3,3,4, \frac{3}{2} \pm \sqrt{9+16 \gamma_{3} / \gamma_{1}}$, which in turn requires that $\gamma_{1}=-2 \gamma_{3}, \quad \gamma_{2}=$ $-\gamma_{3}$. But inconsistency among the four equations in Eq. (7) arises at the $j=2$ level, so that the Painlevé test fails. 
Case 6. $\gamma_{3}=\gamma_{4}=\mathbf{0} ; \mathbf{s}=\mathbf{t}=-\mathbf{2} /\left(\gamma_{1}+\gamma_{2}\right)$

The resonance condition, $\operatorname{det} Q_{j}=0$, leads to the following solutions;

$$
j=-1,0,0,3,3,4, \frac{3}{2} \pm \frac{1}{2\left(\gamma_{1}+\gamma_{2}\right)} \sqrt{25 \gamma_{1}^{2}+18 \gamma_{1} \gamma_{2}-7 \gamma_{2}^{2}} .
$$

The integer resonances occur if (i) $\gamma_{2}=3 \gamma_{1}$, or (ii) $\gamma_{2}=-\gamma_{1}$. The first case (i) leads to inconsistencies among four equations in Eq. (7) at $\mathrm{j}=2$, while the second case (ii) similarly leads to inconsistency at $j=0$. Therefore, the Painlevé test fails in this case.

$$
\text { Case 7. } \gamma_{\mathbf{3}}=\gamma_{\mathbf{4}}=\mathbf{0} ; \gamma_{\mathbf{1}}=\gamma_{\mathbf{2}} ; \mathbf{t}+\mathbf{s}=\mathbf{- 2}
$$

This case corresponds to the well-known integrable vector NLS equation considered by Zakharov and Schulman [A. Together with the parameters; $\gamma_{1}=\gamma_{2}, \gamma_{3}=\gamma_{4}=0$, Eq. (雨) reduces to

$$
2+\gamma_{1}\left(T_{0} U_{0}+R_{0} S_{0}\right)=0 .
$$

The resonances are $j=-1,0,0,0,3,3,3,4$, and it has been checked that the proper number of arbitrary functions exist. Thus, this case passes the Painlevé test.

So far, we have considered the case where $\beta=1$ in Eq. (2). For $\beta=-1$, using the notion of the degenerate dispersion law, Zahkarov and Schulmann found another integrable theory with anomalous dispersive term [7. The Painlevé analysis for the $\beta=-1$ case can be done in the same way as for the $\beta=1$ case. Thus, we suppress the details of analysis and simply state the results. The leading order equation is given by

$$
\begin{aligned}
\gamma_{1} s+\gamma_{2} t-2+\gamma_{4} x & =-\gamma_{3} \frac{t y}{x}, \\
\gamma_{1} s+\gamma_{2} t-2+\gamma_{4} y & =-\gamma_{3} \frac{t x}{y}, \\
\gamma_{1} t+\gamma_{2} s+2+\gamma_{4} x & =-\gamma_{3} \frac{s y}{x} \\
\gamma_{1} t+\gamma_{2} s+2+\gamma_{4} y & =-\gamma_{3} \frac{s x}{y},
\end{aligned}
$$

whose solutions can be grouped into five distinct cases;

(case 1) $x=y$

(case 2) $\gamma_{4}=0, x=-y, \gamma_{3}=\gamma_{1}+\gamma_{2}$

(case 3) $\gamma_{4}=0, x=-y, t=-s$

(case 4) $\gamma_{3}=\gamma_{4}=0, \gamma_{1}=-\gamma_{2}$

(case 5) $\gamma_{3}=\gamma_{4}=0, t=-s$.

Here, only the case 4 passes the Painlevé test. In this case, $S=\left(T_{0} U_{0}-2\right) / R_{0}$ and resonances are $j=$ $-1,0,0,0,3,3,3,4$. This is the integrable system found by Zakharov and Schulmann [4]. All other cases lead to inconsistencies at $j=1$ level thus failing the Painlevé test.

\section{HIROTA BILINEARIZATION AND SOLITONS}

One of the main result of the Painlevé test is to find a new case of coupled NLS equation in Eq. (2) with parameters given by $\gamma_{2}=2 \gamma_{1}, \gamma_{3}=-\gamma_{1}$ and $\gamma_{4}$ arbitrary. With an appropriate scaling, we can always set the nonzero $\gamma_{1}$ to one. Also, as we show in Sec. 4, we can set $\gamma_{4}$ to zero. From now on, we restrict to this case $\left(\beta=1, \gamma_{1}=1, \gamma_{2}=2, \gamma_{3}=-1, \gamma_{4}=0\right)$ and analyze its solution and integrability structures. It is well known that the Painlevé analysis in the preceding section can be related to the Bäcklund transformation (BT). In order to derive 
the BT, we truncate the series in Eq. (3) up to a constant level term and substitute $(z-\psi)$ by an arbitrary function $\phi(z, \bar{z})$ to be determined later. Then, the corresponding $\mathrm{BT}$ is given by

$$
q_{1}=\frac{R_{0}}{\phi}+R_{1}, \quad q_{2}=\frac{T_{0}}{\phi}+T_{1},
$$

where the set $\left(R_{1}, T_{1}\right)$ is a known solution of the coupled NLS equations, which we assume to be the trivial solution $R_{1}=T_{1}=0$. In order for the new set $\left(q_{1}, q_{2}\right)$ to be also a solution, the following equations should hold [9]

$$
\begin{aligned}
i \phi \bar{D} R_{0} \cdot \phi & =i \phi D^{2} R_{0} \cdot \phi-R_{0} D^{2} \phi \cdot \phi+R_{0}^{2} R_{0}^{*}+2 R_{0} T_{0} T_{0}^{*}-R_{0}^{*} T_{0}^{2} \\
i \phi \bar{D} T_{0} \cdot \phi & =i \phi D^{2} T_{0} \cdot \phi-T_{0} D^{2} \phi \cdot \phi+T_{0}^{2} T_{0}^{*}+2 T_{0} R_{0} R_{0}^{*}-T_{0}^{*} R_{0}^{2}
\end{aligned}
$$

Here, the Hirota's bilinears $D$ and $\bar{D}$ are defined by

$$
\bar{D}^{n} D^{m} f \cdot g=\left.\left(\frac{\partial}{\partial \bar{z}}-\frac{\partial}{\partial \bar{z}^{\prime}}\right)^{n}\left(\frac{\partial}{\partial z}-\frac{\partial}{\partial z^{\prime}}\right)^{m} f(z, \bar{z}) g\left(z^{\prime}, \bar{z}^{\prime}\right)\right|_{\substack{z=z^{\prime} \\ \bar{z}=\bar{z}^{\prime}}}
$$

Equation (20) can be decoupled as

$$
\begin{aligned}
R_{0} D^{2} \phi \cdot \phi-\left(\gamma_{1} R_{0}^{2} R_{0}^{*}+\gamma_{2} R_{0} T_{0} T_{0}^{*}+\gamma_{3} R_{0}^{*} T_{0}^{2}\right) & =\lambda_{1} R_{0} \phi \cdot \phi, \\
T_{0} D^{2} \phi \cdot \phi-\left(\gamma_{1} T_{0}^{2} T_{0}^{*}+\gamma_{2} T_{0} R_{0} R_{0}^{*}+\gamma_{3} T_{0}^{*} R_{0}^{2}\right) & =\lambda_{2} T_{0} \phi \cdot \phi, \\
i \bar{D} R_{0} \cdot \phi & =D^{2} R_{0} \cdot \phi-\lambda_{1} R_{0} \cdot \phi, \\
i \bar{D} T_{0} \cdot \phi & =D^{2} T_{0} \cdot \phi-\lambda_{2} T_{0} \cdot \phi .
\end{aligned}
$$

Now, explicit N-solitons can be constructed in the usual way by solving $\phi, R_{0}, T_{0}$ in terms of power series.

\section{A. one-soliton}

For one soliton solution, we choose $\lambda_{1}=\lambda_{2}=0$ and assume solutions in a series form in $\epsilon$ such that $\phi=1+\epsilon^{2} h, R_{0}=$ $\epsilon R, T_{0}=\epsilon T$. Then, by equating the coefficients of the polynomials to zero in Eq. (22) and solving them explicitly, we obtain

$$
R=\alpha \exp \left(i\left(a^{2}-b^{2}\right) \bar{z}+2 a b \bar{z}+i a z+b z\right), \quad T=\beta \exp \left(i\left(a^{2}-b^{2}\right) \bar{z}+2 a b \bar{z}+i a z+b z\right),
$$

where $\alpha, \beta$ are arbitrary complex numbers while $a, b$ are arbitrary real numbers. $h$ is also obtained by solving the third order equation such that

$$
h=\frac{1}{8 b^{2}}\left(|\alpha|^{2}+2|\beta|^{2}-\frac{\alpha^{*} \beta^{2}}{\alpha}\right) \exp (2 b z+4 a b \bar{z}) .
$$

Consistency requires that phases of the complex numbers $\alpha$ and $\beta$ should be either the same, or differ by $\pi / 2$. In the case of the same phase, we parameterize $\alpha$ and $\beta$ by

$$
\alpha=\sqrt{8} b \cos k e^{\Delta+i \theta}, \quad \beta=\sqrt{8} b \sin k e^{\Delta+i \theta},
$$

in terms of arbitrary real numbers $k, \theta, \Delta$. Then, the final form of the one soliton solution is given by substituting $\epsilon=1$ in Eq. (19) such that

$$
\begin{aligned}
& q_{1}=\sqrt{2} b \cos k e^{i\left(a^{2}-b^{2}\right) \bar{z}+i a z+i \theta} \operatorname{sech}(b z+2 a b \bar{z}+\Delta), \\
& q_{2}=\sqrt{2} b \sin k e^{i\left(a^{2}-b^{2}\right) \bar{z}+i a z+i \theta} \operatorname{sech}(b z+2 a b \bar{z}+\Delta) .
\end{aligned}
$$

In the case where phases differ by $\pi / 2, \alpha$ and $\beta$ are given by

$$
\beta= \pm i \alpha= \pm i \sqrt{8} b e^{\Delta+i \theta} .
$$

Then, the corresponding one-soliton solution is

$$
\begin{aligned}
& q_{1}=\sqrt{2} b e^{i\left(a^{2}-b^{2}\right) \bar{z}+i a z+i \theta} \operatorname{sech}(b z+2 a b \bar{z}+\Delta) \\
& q_{2}= \pm i \sqrt{2} b e^{i\left(a^{2}-b^{2}\right) \bar{z}+i a z+i \theta} \operatorname{sech}(b z+2 a b \bar{z}+\Delta) .
\end{aligned}
$$




\section{B. two-soliton}

The two-soliton solution can be obtained using the series expansion $\phi=1+\epsilon^{2} h_{1}+\epsilon^{4} h_{2}, \quad R_{0}=\epsilon \rho_{1}+\epsilon^{3} \rho_{2}, \quad T_{0}=$ $\epsilon \tau_{1}+\epsilon^{3} \tau_{2}$. Inserting these ansätze into Eq. (22), we obtain solutions

$$
\begin{aligned}
& \rho_{1}=f+g, \tau_{1}=i \rho_{1} ; f \equiv e^{-i k^{2} \bar{z}+k z+\eta_{f}}, g \equiv e^{-i l^{2} \bar{z}+l z+\eta_{g}}, \\
& h_{1}=2\left(\frac{f f^{*}}{\left(k+k^{*}\right)^{2}}+\frac{f g^{*}}{\left(k+l^{*}\right)^{2}}+\frac{g f^{*}}{\left(l+k^{*}\right)^{2}}+\frac{g g^{*}}{\left(l+l^{*}\right)^{2}}\right),
\end{aligned}
$$

where $k, l, \eta_{f}, \eta_{g}$ are arbitrary complex numbers. Also, after a lengthy but straightforward calculation we obtain

$$
\begin{aligned}
& \rho_{2}=2(l-k)^{2}\left(\frac{f f^{*} g}{\left(k+k^{*}\right)^{2}\left(l+k^{*}\right)^{2}}+\frac{f g g^{*}}{\left(k+l^{*}\right)^{2}\left(l+l^{*}\right)^{2}}\right), \quad \tau_{2}=i \rho_{2}, \\
& h_{2}=\frac{4(l-k)^{2}\left(l^{*}-k^{*}\right)^{2} f f^{*} g g^{*}}{\left(k+k^{*}\right)^{2}\left(l+k^{*}\right)^{2}\left(k+l^{*}\right)^{2}\left(l+l^{*}\right)^{2}} .
\end{aligned}
$$

Finally, the two-soliton solution is obtained by taking $\epsilon=1$ in the BT equation $q_{1}=\frac{R_{0}}{\phi}, q_{2}=\frac{T_{0}}{\phi}$.

Surprisingly, there exists a different type two-soliton solution which can be obtained by a simple linear superposition of the left-polarized one-soliton with the right-polarized one-soliton;

$$
q_{1}=\frac{f}{\phi_{1}}+\frac{g}{\phi_{2}}, \quad q_{2}=i \frac{f}{\phi_{1}}-i \frac{g}{\phi_{2}},
$$

where $\phi_{1}=1+2 f f^{*} /\left(k+k^{*}\right)^{2}, \phi_{2}=1+2 g g^{*} /\left(l+l^{*}\right)^{2}$. The reason underlying the existence of such a linear superposition is explained in the following section.

\section{INTEGRABILITY}

The Painlevé test in Sec. 2 suggests new integrable cases of coupled NLS equations. As we have shown in the preceding section, the coupled NLS equation with $\gamma_{1}=1, \gamma_{2}=2, \gamma_{3}=-1, \gamma_{4}=0$ possesses exact soliton solutions, which reflects the integrability of the equation. Before proving the integrability by deriving the corresponding Lax pair, we first note that taking $\gamma_{4}=0$ is not essential. Make a change of variables such that

$$
Q_{1}=x q_{1}+y q_{2}, \quad Q_{2}=y q_{1}+x q_{2}
$$

If $\left(Q_{1}, Q_{2}\right)$ satisfy the coupled NLS equation in Eq. (2) with $\gamma_{1}=1, \gamma_{2}=2, \gamma_{3}=-1, \gamma_{4}=0$, then $\left(q_{1}, q_{2}\right)$ satisfy Eq. (2) but with parameters $\gamma_{1}=1, \gamma_{2}=2, \gamma_{3}=-1, \gamma_{4}=\frac{4 x y}{x^{2}+y^{2}}$. Thus, we set $\gamma_{4}$ to zero without loss of generality. The integrability and the Lax pair of the coupled NLS equation in Eq. (2) with $\gamma_{1}=1, \gamma_{2}=2, \gamma_{3}= \pm 1, \gamma_{4}=0$ follows from the observation that these equations can be embeded in the integrable coupled NLS equation based on the symmetric space $S p(2) / U(2)$ given by!

$$
\begin{aligned}
i \bar{\partial} \psi_{1} & =\left[\partial^{2} \psi_{1}+2 \psi_{1}^{2} \psi_{1}^{*}+4 \psi_{1} \psi_{2} \psi_{2}^{*}+2 \psi_{2}^{2} \psi_{3}^{*}\right] \\
i \bar{\partial} \psi_{2} & =\left[\partial^{2} \psi_{2}+2 \psi_{2} \psi_{1} \psi_{1}^{*}+2 \psi_{2}^{2} \psi_{2}^{*}+2 \psi_{3} \psi_{1} \psi_{2}^{*}+2 \psi_{3} \psi_{2} \psi_{3}^{*}\right] \\
i \bar{\partial} \psi_{3} & =\left[\partial^{2} \psi_{3}+2 \psi_{3}^{2} \psi_{3}^{*}+4 \psi_{3} \psi_{2} \psi_{2}^{*}+2 \psi_{2}^{2} \psi_{1}^{*}\right] .
\end{aligned}
$$

Consistent reductions can be made if we take $\psi_{1}= \pm \psi_{3}$, which are precisely the cases $\gamma_{1}=2, \gamma_{2}=4, \gamma_{3}= \pm 2, \gamma_{4}=0$ in Eq. (2). Furthermore, Eq. (33) arises from the Lax pair

$$
L_{z} \Psi \equiv[\partial+E+\lambda T] \Psi=0, \quad L_{\bar{z}} \Psi \equiv\left[\bar{\partial}+\left(\frac{1}{2}[E, \tilde{E}]-\partial \tilde{E}\right)-\lambda E-\lambda^{2} T\right] \Psi=0
$$

\footnotetext{
${ }^{1}$ The generalization of NLS equation using symmetric spaces and the concept of matrix potential can be found in 5.60 .10 . 12 .
} 
where the $4 \times 4$ matrices $E$ and $T$ are

$$
E=\left(\begin{array}{cccc}
0 & 0 & \psi_{1} & \psi_{2} \\
0 & 0 & \psi_{2} & \psi_{3} \\
-\psi_{1}^{*} & -\psi_{2}^{*} & 0 & 0 \\
-\psi_{2}^{*} & -\psi_{3}^{*} & 0 & 0
\end{array}\right), T=\left(\begin{array}{cccc}
i / 2 & 0 & 0 & 0 \\
0 & i / 2 & 0 & 0 \\
0 & 0 & -i / 2 & 0 \\
0 & 0 & 0 & -i / 2
\end{array}\right)
$$

By taking $\psi_{1}= \pm \psi_{3}$ in Eqs. (34) and (35), we obtain the Lax pair for the coupled NLS equation in Eq. (2) with $\gamma_{1}=2, \gamma_{2}=4, \gamma_{3}= \pm 2, \gamma_{4}=0$.

More directly, the integrability can be shown by mapping the coupled NLS equation into two independent (decoupled) NLS equations as follows; if we substitute

$$
\Psi_{1}=q_{1}+i q_{2}, \quad \Psi_{2}=q_{1}-i q_{2},
$$

in the two independent NLS equations, $i \bar{\partial} \Psi_{k}=\partial^{2} \Psi_{k}+2\left|\Psi_{k}\right|^{2} \Psi_{k} ; k=1,2$, we recover Eq. (2) with $\gamma_{1}=2, \gamma_{2}=$ 4, $\gamma_{3}=-2, \gamma_{4}=0$. Similarly using the substitution $\Psi_{1}=q_{1}+q_{2}, \Psi_{2}=q_{1}-q_{2}$, we obtain Eq. (2) with $\gamma_{1}=2, \gamma_{2}=$ $4, \gamma_{3}=2, \gamma_{4}=0$. This explains why the linear superposition of two solitons was possible in the previous section. The decomposition of the coupled NLS equation into two independent NLS equations implies that the linear combination of solutions according to Eq. (36) becomes a solution of the coupled NLS equation. Group theoretically, such a decomposition corresponds to the embedding of symmetric spaces, $(S U(2) / U(1)) \times(S U(2) / U(1)) \subset S p(2) / U(2)$. According to the group theoretic construction of the NLS equation using Hermitian symmetric spaces [13], the above embedding results in two decoupled NLS equations. It is interesting to see that this decoupling behavior is also reflected in the Painlevé analysis. Besides the solution of the leading order equation (4) (the case 4 in Sec. 2) which enables the present coupled NLS equation to pass the Painlevé test, for the set of parameters $\gamma_{2}=2 \gamma_{1}, \gamma_{3}=-\gamma_{1}$, we have another set of solutions of the leading order equation (4),

$$
U_{0}=\frac{-2 T_{0}}{T_{0}^{2}+R_{0}^{2}}, \quad S_{0}=\frac{-2 R_{0}}{T_{0}^{2}+R_{0}^{2}}
$$

This has resonances at $j=-1,-1,0,0,3,3,4,4$. This solution also passes the test. Note that all resonances are double poles and each poles are precisely those of the NLS equation. This suggests that the systems under consideration are indeed two independent NLS systems.

So far, we have restricted to the case $\beta=1$. For $\beta=-1$, our Painleve analysis showed that the only integrable case is the vector NLS equation considered by Zakharov and Schulmann,

$$
i \bar{\partial} \Psi=\partial^{2} \Psi+\Psi \xi \Psi, \quad-i \bar{\partial} \xi=\partial^{2} \xi+\xi \Psi \xi
$$

where $\Psi=\left(\psi_{1}, \psi_{2}\right)$ and $\xi=\left(\chi_{1}, \chi_{2}\right)$. Using the reduction $\xi=\Psi^{*} A$ with $A=\left(\begin{array}{cc}-1 & 0 \\ 0 & 1\end{array}\right)$ and substituting $q_{1}=$ $\psi_{1}, q_{2}=\psi_{2}^{*}$, one can recover the vector NLS equation as in Eq. (2) with $\beta=-1, \gamma_{1}=-\gamma_{2}, \gamma_{3}=\gamma_{4}=0$.

In a similar vein, we construct a new integrable equation with $\beta=-1$ which resembles the previous decoupling NLS equation with $\beta=1$. We take

$$
M=\left(\begin{array}{cc}
\chi_{1} & \chi_{2} \\
\chi_{2} & -\chi_{1}
\end{array}\right), \quad N=\left(\begin{array}{cc}
-\chi_{1}^{*} & \chi_{2}^{*} \\
\chi_{2}^{*} & \chi_{1}^{*}
\end{array}\right)
$$

and define the coupled NLS equation by

$$
\begin{aligned}
i \bar{\partial} M & =\partial^{2} M-2 M N M, \\
-i \bar{\partial} N & =\partial^{2} N-2 N M N .
\end{aligned}
$$

We find that Eq. (40) arises from the Lax pair $\left(\left[L_{z}, L_{\bar{z}}\right]=0\right)$

$$
\begin{aligned}
L_{z} & =\partial+\left(\begin{array}{cc}
0 & M \\
N & 0
\end{array}\right)+i \frac{\lambda}{2}\left(\begin{array}{cc}
I_{2 \times 2} & 0 \\
0 & -I_{2 \times 2}
\end{array}\right), \\
L_{\bar{z}} & =\bar{\partial}-i\left(\begin{array}{cc}
0 & \partial M \\
-\partial N & 0
\end{array}\right)-i\left(\begin{array}{cc}
M N & 0 \\
0 & -N M
\end{array}\right)-\lambda\left(\begin{array}{cc}
0 & M \\
N & 0
\end{array}\right)-\frac{i}{2} \lambda^{2}\left(\begin{array}{cc}
I_{2 \times 2} & 0 \\
0 & -I_{2 \times 2}
\end{array}\right) .
\end{aligned}
$$

If we substitute $q_{1}=\chi_{1}, q_{2}=\chi_{2}^{*}$, we have an integrable equation with anomalous dispersion term and asymmetric coupling,

$$
\begin{aligned}
& i \bar{\partial} q_{1}=\partial^{2} q_{1}+2\left(\left|q_{1}\right|^{2} q_{1}-2\left|q_{2}\right|^{2} q_{1}-q_{2}^{* 2} q_{1}^{*}\right), \\
& i \bar{\partial} q_{2}=-\partial^{2} q_{2}+2\left(\left|q_{2}\right|^{2} q_{2}-2\left|q_{1}\right|^{2} q_{2}-q_{1}^{* 2} q_{2}^{*}\right) .
\end{aligned}
$$

This equation does not belong to the coupled NLS equation in Eq. (2) which has been Painlevé tested. 


\section{DISCUSSION}

In this paper, we have performed a Painlevé analysis for coupled NLS equations with coherent coupling terms as given in Eq. (2). Besides the well-known vector NLS equation $\left(\beta= \pm 1 ; \gamma_{2}= \pm \gamma_{1}, \gamma_{3}=\gamma_{4}=0\right)$, we have found new integrable cases which are defined by the set of parameters with $\beta=1$; (i) $\gamma_{2}=2 \gamma_{1}, \gamma_{3}=-\gamma_{1}, \gamma_{4}$ arbitrary , or (ii) $\gamma_{2}=2 \gamma_{1}, \gamma_{3}=\gamma_{1}, \gamma_{4}=0$. Painlevé analysis shows that these are the only integrable cases except the vector NLS equation. We have shown that these new equations are essentially identical to two independent sets of NLS equations. Physically, the first case describes the propagation of optical pulses in an isotropic nonlinear medium in which the third-order susceptibility tensor satisfies that $\chi_{x x y y}^{(3)}+\chi_{x y x y}^{(3)}=-2 \chi_{x y y x}^{(3)}$, while the second case does not have a similar interpretation. The linear transformation in Eq. (36), which decouples the interacting NLS equation (case (i)) into two independent NLS equations, also maps two orthogonal linearly polarized lights into the left and the right circularly polarized lights. Thus, in such an isotropic medium, left and right circularly polarized lights do not interact each other thereby preserving circular polarizations. This case may be compared with a polarization preserving fiber where only one particular polarization direction is preserved. It would be interesting to know whether there exists nonlinear isotropic materials possessing this property.

\section{ACKNOWLEDGEMENT}

This work was supported in part by Korea Science and Engineering Foundation (KOSEF) 971-0201-004-2/97-07-0202-01-3, and by the program of Basic Science Research, Ministry of Education BSRI-97-2442, and by KOSEF through CTP/SNU.

[1] see for example, M.N. Islam, Ultrafast Fiber Switching Devices and Systems (Cambridge Press, New York, 1992).

[2] G.P. Agrawal, Nonlinear Fiber Optics (Academic Press, New York, 1995).

[3] V. E. Zakharov and A.B. Shabat, Funct. Anal. Appl. 8, 43 (1974).

[4] V. E. Zakharov and E. I. Schulman, Physica 1D 192 (1980).

[5] A.P. Fordy and P.P. Kulish, Commum. Math. Phys. 89, 427 (1983);P. Oh and Q-H. Park, Phys. Lett. 383B, 333 (1996).

[6] Q-H. Park, H.J. Shin, and Jongbae Kim, SNUTP 97-158, submitted to Commun. Math. Phys.; Jongbae Kim, Q-H. Park, and H.J. Shin, to be published in Phys. Rev, E.

[7] V. E. Zakharov and E. I. Schulman, Physica 4D 270 (1982).

[8] J. Weiss, M. T. Tabor, and G. Carnevale, J. Math. Phys. 24, 552 (1983).

[9] R. Sahaderan, K.M. Tamizhmani, and M. Lakshmanan, J. Phys. A 19, 1783 (1986).

[10] I. Bakas, Q-Han Park and H. J. Shin, Phys. Lett. B372, 45 (1996).

[11] Q-H. Park and H.J. Shin, J. Korean. Phys. Soc. 30, 336 (1997).

[12] Q-H. Park and H.J. Shin, Phys. Rev. A57 ,4621 (1998), ibid A57 , 4643 (1998).

[13] S. Helgason, Differential geometry, Lie groups and symmetric spaces. 2nd ed. New York; Academic Press 1978. 